\title{
Efficacy of Social Media Communications for Enhancing Student Success
}

\section{Dr. Louis J. Everett, University of Texas, El Paso}

Dr. Everett is the MacGuire Distinguished Professor of Mechanical Engineering at the University of Texas El Paso. Dr. Everett's current research is in the areas of Mechatronics, Freshman Programs and Student Engagement. Having multiple years of experience in several National Laboratories and Industries large and small, his teaching brings real world experiences to students. As a former NSF Program Director he works regularly helping faculty develop strong education proposals.

\section{Dr. Norman Love, University of Texas, El Paso}

Norman D. Love, Ph.D.is an Associate Professor in the Department of Mechanical Engineering. Dr. Love, an El Paso native and UTEP alumnus, earned a B.S. and M.S. in Mechanical Engineering from the University of Texas El Paso and completed his Ph.D. at the University of Oklahoma in the same field. Dr. Love's research interests lie in the areas of combustion and energy conversion processes and engineering education. He has developed flipped classroom modules and also implements project based learning activities in his class activities.

\section{Md Moinuddin Shuvo, University of Texas at El Paso}

Md Moinuddin Shuvo is a graduate student at the University of Texas at El Paso with a background in Mechanical Engineering. He pursued his Bachelor from Bangladesh University of Engineering and Technology in the same subject. His research interest lies in Additive Manufacturing, Fracture Mechanics, Powder Metallurgy. He also likes to engage with students to help them learn better as he pursues a career in research-based academia while working as a teaching assistant at his graduate school.

\section{Mr. Vishal Bhimrao Zade, University of Texas, El Paso}

Vishal Zade is a PhD Candidate in Mechanical Engineering at University of Texas, El Paso. He has been working on his research with bulk ceramics and helping students as a Teaching Assistant. 


\section{Efficacy of Social Media Communications for Enhancing Student Success - Preliminary Results}

Thermodynamics is a difficult course for many Undergraduate students. At the University of Texas at El Paso it is no different. The mechanical engineering department teaches

Thermodynamics as a first term junior course. There is unfortunately a large failure rate for the class.

The University of Texas at El Paso (UTEP) is the second oldest academic institution in the University of Texas System. UTEP is located in one of the largest bi-national communities in the world and is unique among research institutions. UTEP is home to a majority Hispanic student population, many of who are among the first in their families to attend college. The University's commitment to providing both access and excellence is reflected in the unique demography of its student body - a $21^{\text {st }}$ Century demography that reflects its service area in terms of ethnicity and gender. Engineering has been at the heart of UTEP since its origin. The College ethnicity reflects the service area (majority Hispanic - 81\%); increasing the participation of females in engineering is a work in progress. It is notable that the College has been successful in attracting minority women into engineering with the current female population $(\sim 20 \%)$ being primarily Hispanics.

The intervention described in this paper uses an on-line communication software that can be thought of as a form of "social media". According to Margaret Rouse of Whatis.com "Social media is the collective of online communications channels dedicated to community-based input, interaction, content-sharing and collaboration." This paper uses a software that allows students to provide input, interact, share and collaborate, hence we refer to the intervention as a social media. The work being reported speaks to the efficacy of social media as defined by Rouse and not dependent on the software medium. As a result, we do not identify the software but only give results of its use.

Although literature on the use of social media and its proper use to improve on student learning in the classroom is somewhat limited, engagement and learning done by students in an online or blended environment has been researched for several years with its popularity significantly growing over the last 10 years [1-7]. This includes studies investigating the use of online tools and their impact on student learning [1]. Wallace investigated the interactions among teachers and students in an online learning environment and recommended future research be done on how social, personal, and interpersonal aspects are related to learning [2]. Rovai and Jordan have shown that blended learning produces a stronger sense of community among students compared to a completely traditional or online course [3]. There have even been studies done to investigate the impact of web logging, better known as blogging, on student learning. Williams found that blogging implemented in the classroom has the potential to be a transformational technology for teaching and learning [4]. Davies and Graff studied the frequency of online interactions when using a software and found that online interactions did not lead to significantly higher performance for students achieving passing grades [5].

As mentioned previously, the intervention reported in this paper helps students take ownership of their education using online social media software. Harrison and Thomas investigated the use of 
social networking sites on learning for language learners. The authors found that longitudinal studies are required to observe the impact of the tool on learning [8]. Brady et al. investigated the use of social networking software, Ning, in the classroom. The study showed that educationbased social networks are most effective for distance education courses for improved online communications [9]. Hung and Yuen studied the use of social networking in a face-to-face course [10]. Findings showed that a majority of participants developed feelings of social connectedness and were more favorable to the course when social networking was used. Work by Chan et. al. [11] demonstrated that "ownership", or taking an active role in the educational process yields several advantages. Schroeder et al. [12] found that using social media increases student engagement, provides a platform to showcase work, and maintains learning communities. Tess [13] conducted a study of social media use in classrooms and found that its effectiveness is still being questioned. Although previous studies have shown the use of social media software in the classroom is overall beneficial, there still seems to be many questions regarding how to effectively use social media in the classroom for improved student learning or increased passing rates.

This paper describes an experiment and its results. Students enrolled in a Thermodynamics class used a social media software to interact with each other and the instructor/TA. At the beginning of the semester the students were introduced to the platform as a means of communication with their fellow classmates. The motivation presented to the students was that by helping each other, a deeper understanding of content would be possible. Student activity was monitored daily on the platform to make sure students did not post inappropriate content, materials that could be seen as documents for cheating, or engage in highly negative feedback to each other. Students who were violating the ethical rules were informed and any post was immediately removed. We observed little student activity on the site during the first half of the semester. During this lightly used time, the class took two major exams. After the second major exam the students were encouraged to use the platform by giving them a very minor grade incentive in the form of extra credit. This incentive caused some students to begin using the software very significantly. During this incentive period, the class was given another two major exams. Data collected on exam performance and how much and how effectively the communication was used was collected and analyzed to determine if the software has an impact on student performance.

Students were introduced to the software at the beginning of the term but it was not emphasized. In the first half of the semester, the students took two major in-class, individual exams. These exams formed the "initial" performance. After the second exam, the instructor offered a maximum of 2 points for significant activity with the software. After this announcement, the students took another two major in-class, individual exams. The average of these two formed the final performance. From the initial and the final we computed improvement or decline.

When students answered questions, they can be "up voted" by fellow students and faculty. Up voted students earn more credit. The maximum extra credit is 2 points out of 100 . This is not much but is a little encouragement for them to help others by helping themselves. In the long term, should the methods prove successful, by maintaining longitudinal data, students can use the up votes from their complete undergraduate experience to produce portfolio evidence they can be proud to show potential employers and graduate schools. This can provide a quantifiable measure of student performance independent of grades. The University of Texas at El Paso consists of predominately commuter students who hold off campus jobs. The typical student 
comes to campus for class and quickly leaves campus for work. The university has created a number of incentives to encourage students to remain on campus and interact with faculty and students. These efforts have had marginal success. The ultimate purpose therefore is to investigate the efficacy in using "remote", "on-line" software to facilitate seeking help when the person cannot participate in face to face interactions.

Although it may be true that social media can be a significant distraction, the software provider claims this platform was designed to encourage student interaction and discourage distractions. Since social media is already an integral part of a student's day, using it to study is expected to be welcomed by them. Nonetheless, it is true this experiment had a dedicated team of Professors, TAs and Tutors on the platform to monitor questions and respond. Questions that needed further deliberation were taken up in class or office hours. It would be interesting to see the impact of less instructor moderation.

Hypothesis:

Thermodynamics is a class with the highest failure rate in the mechanical engineering department at UTEP. To mitigate the attrition of graduating class the given year, a range of techniques to improve passing rates have been implemented. These include online available materials, clicker questions, and other similar interventions. Unfortunately, these interventions have not had a significant impact in the overall passing rate for the course. Given the extent of influence social media, a new strategy was devised with the goal of improving the passing rate of the class.

As a pilot project, one class with the highest failure rate in the Mechanical Engineering Department, Thermodynamics, was chosen to implement a social media-based communication platform. The study investigates the following:

- The effect of an incentive on student participation in the social media platform. The first half of the semester introduced the software with no grade incentive while the second half of the semester included a grade incentive for participation.

- The effect of activity and engagement on the social media platform on the final grade

The online communications software is a platform designed specifically for college classes. At the beginning of the semester at UTEP this platform was introduced by the professor to students, as a tool for their communication with each other and the faculty member. A total of 95 students were enrolled in the class. Only 77 joined the software platform. Of these 77 enrolled users 18 neither asked nor answered any questions. It is possible these 18 read posts of others but we did not track that activity. A student can join the website only by using their institutional email ID ensuring the website service is exclusive to them and to prevent illegal access. The online platform has a motto of making the learning procedure of the students more collaborative. When the students were first introduced to the website, the instructor made clear that the purpose of the website was to help them throughout the learning procedure. The instructor told students to mainly ask thoughtful questions about the subject, but light hearted questions to release tension before exams were also welcome. Some of the features of the platform are as follows- Students had the option of asking questions anonymously or with their own username. They could also select a specific test or homework assignment to ask a question. General questions could also be 
asked. The questions asked by the students can be seen by all the other students enrolled in the same classroom. Anyone can answer the questions and upon answering and receiving votes (similar feature to Facebook) students earn points.

Some of the example questions taken from the thermodynamic course are as followsStudent 1 -what is the formula to get thermal efficiency?

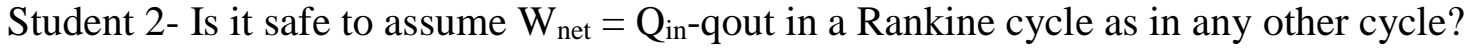

Student 3- Can we assume $\mathrm{v}_{1}=\mathrm{v}_{2}$ and $\mathrm{P}_{2}=\mathrm{P}_{3}$ in a standardize ranking cycle?

Student 4- Will there be a review session for the Final Exam?

As it can be seen from the questions asked that most of the questions were related based on the topic and were relevant but it should also be addressed that some of the questions were general queries or posts before an exam is approaching to lighten the mood of the class and the Instructor and the TAs made sure that these questions did not outnumber the subject related questions.

Data that were required for the study were generated and provided by the social media software development team (a private company with no connection to the university) in accordance with the study. The data generated was mainly based on student's activity on the site such as number of questions asked, number of questions answered, number of up votes received, and student's reputation points assigned by the software company algorithm.

Assessment parameters:

Two assessment parameters were identified to measure the student's engagement on the platform and how well they collaborated with each other. These parameters were as follows-

- Level of activity (measured using questions student asked and answered)

- Level of engagement (measured using “up" votes received)

The students were instructed to ask questions related to the subject as much as possible but interactive posts were also encouraged to make the platform a friendly environment for communication to grow. As such, the number of questions asked by each student were set as a level of activity parameter. In addition, questions answered by the students were also set as a level of activity parameter because some students were in their advanced level of understanding of the subject and preferred to answer questions more compared to asking questions on the platform.

Another assessment parameter for the student's engagement on the platform was the level of engagement which was translated from the so-called "up" votes each student received on their questions, answers and comments. The "up" votes can be translated to the level of appreciation from other students as they answer their questions or asked any questions which other students wanted to ask too. As per assumption, the higher the number of "up" votes a student receives the more engaged and larger impact they are thought to have while using the platform with other students.

Limitations of study:

The authors would like to address the limitations regarding this particular study. 
- One subject studied: This particular study was only performed on one subject, Thermodynamics. The authors chose this subject because of the high failure rates among previous classes.

- The data set is based on exam grades, it was the intent of the instructor to maintain the same level of difficulty for exams before and after the social media system was incentivized with extra credit. Future studies will investigate the performance of classes from one semester to the next.

- Limited sample size: The sample size was limited to a single class with approximately 77 participating students.

- For activity and engagement parameters ensuring that the content was relevant was difficult to control over the course of the semester. For examples, one significant comment or even a good joke (unfortunately) can generate up votes from every student in class. Only one up vote per student per post was allowed discouraging one person up voting his friend excessively.

- Students can ask a very obvious or trivial question and it will still be awarded 1 point of participation.

- Unfortunately a comparison to previous semesters where the software was not available cannot be made. This is the first semester the instructor taught this subject, making comparisons between previous semesters would introduce variability in students and instructor. The authors feel any such comparison would be deceptive.

\section{Results and discussion:}

One aspect we were interested in investigating was the willingness for students to help each other. We began with several measures to identify participation and engagement. We then investigated the relationships between the measures to identify which ones were most useful. We collected data on the number of questions asked, the number of questions answered and the number of times the student's question or answer was "up-voted" or "liked". Up-voting we believe is an indication of engagement. Our thinking is if a student is disengaged or unaware of what the class is focused on, their posted questions/answers have low likelihood of being upvoted because they are out of touch with what most students are concerned with. If they are fully engaged with what is current, their interaction is more likely to be considered important, relevant and hence up-voted.

The first comparison we made was to look at activity defined as the number of questions asked plus the number answered and compared that to the number of up-votes (engagement). 


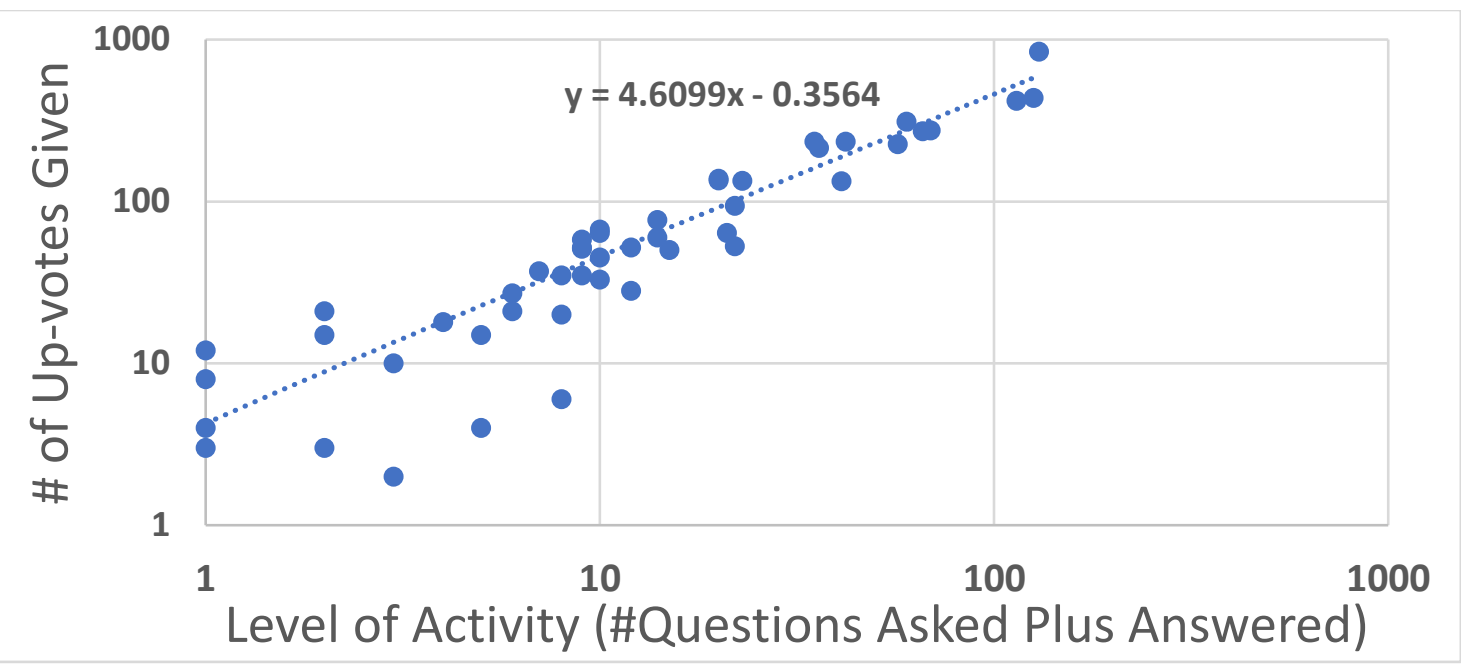

Figure 1 - Engagement (upvotes) versus Activity (\#Questions asked plus answered).

Figure 1 shows the results of students who had an activity of at least 1 . The trend line shows a type of expectation. In general, one should expect that increasing activity will provide increasing engagement. Points lying significantly above the expectation are people whose activity creates excitement. These would be people whose comments are highly valued. The instructor, for example, would be expected to lie well above the expectation line. A student falling below the expectation represents someone whose contribution is not appreciated.

The data shows that there are several students falling significantly below the expectation. These students also had the lower values of activity. Note that by the time students are in the double digits of activity, their engagement is roughly the expected values. One possible explanation is that students who are significantly active are getting up-votes because they are engaged sufficiently to keep abreast of what the class is concerned about. If this is true, many of the lower activity students are not up-voted because they are "out of touch" with what the class is concerned with. Further investigations will look for evidence that higher than expected engagement is indicative of staying in touch with the class. If this is shown to be true, low engagement could be used as an indicator of students who are falling behind.

Another point of interest was whether or not students were taking ownership of their learning. Are the students helping each other? Figure 2 shows the results for all the students. There are several data points falling at the origin representing the students who never asked or answered a single question. Just because these students have 0 activity levels does not mean they received no benefit. They might have been reading the posted questions and answers and benefiting from other active students. Although watching and benefiting they may not have contributed. 


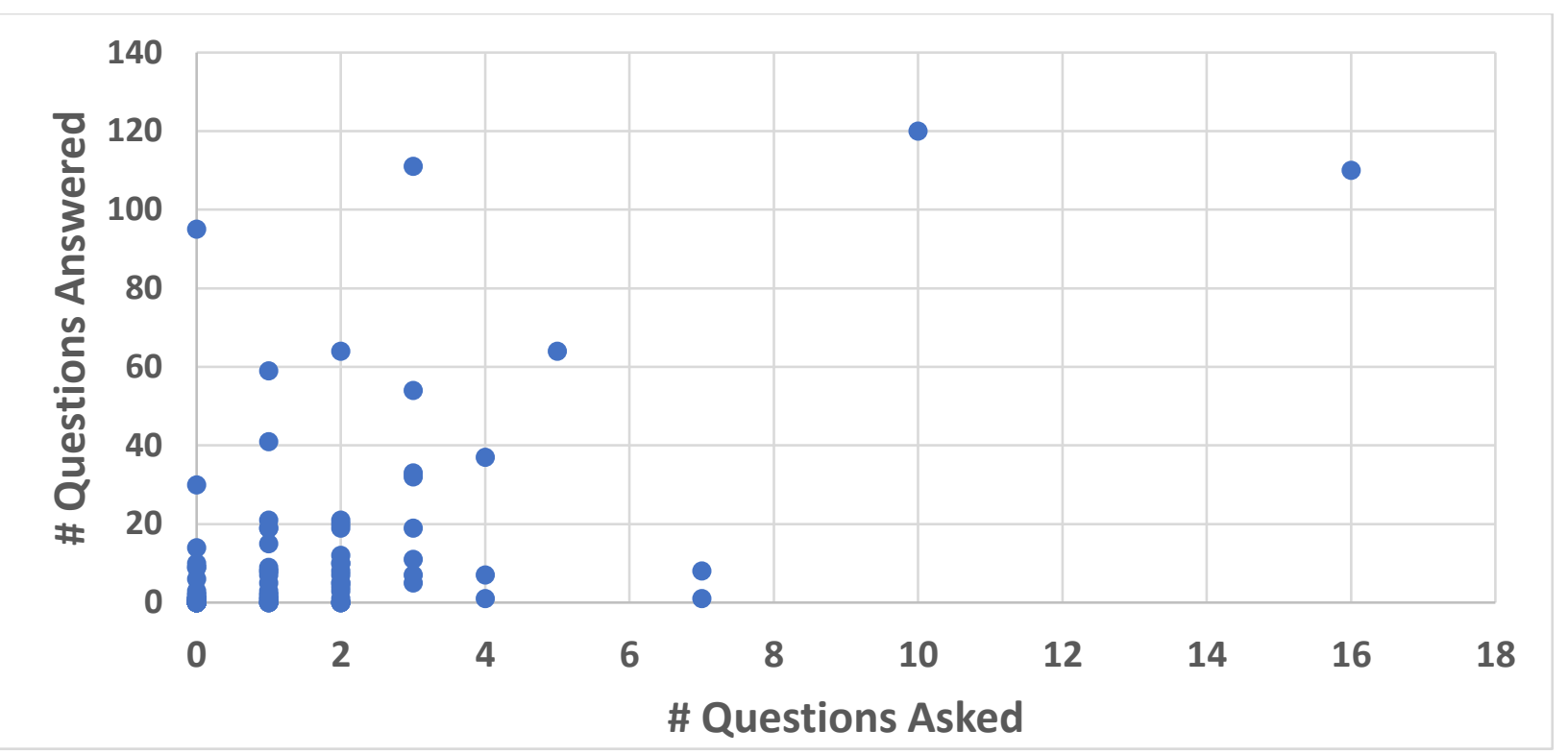

Figure 2 - Questions Asked Answered Versus Questions Asked.

One would expect that an instructor would have significantly more answers than questions. The data shows four students behaving like an instructor. When looking for teaching assistants, data as shown in Figure 2 might be used to identify students with the right attitude for teaching.

As mentioned previously, we also had interest to see if this method of interacting had a positive impact on learning. We introduced the software from the first day of class but did nothing special to encourage students to use it. The students took two exams in the first half of the class. The average of the first two exams was taken as the initial performance. Due to 3 extra points incentive offered to encourage homework, the maximum possible initial performance was 103.

After taking the second exam, the instructor told the students that extra credit would be given for high levels of participation. The instructor did not indicate how many points would be given just that there would be some points. This promise of extra credit increased participation significantly. Unfortunately, one limitation of the study is that we did not collect activity data pre and post the extra credit announcement. We know the activity increased by observing the student behavior, but we did not quantify the increase. Future work will collect data pre and post so the impact of extra incentive can be studied.

After the activity incentive, the students took two more exams. The average of the last two exams became the final performance. We compared the final performance to initial performance to determine the impact of the activity. The instructor tried to keep the level of difficulty uniform over all the exams. There is some evidence that the level of difficulty between the first two and the last two exams was similar because the performance of the non-participating students showed no significant correlation between those whose performance increased and those who decreased (Figure 3).

Shown in Figure 3 are the results for the inactive students. The horizontal axis is the student number, you can easily see that there were 18 students who had a 0 level of activity. For most 
students there are two dots. The blue dot is the initial performance. If the student improved, called improvers, there is a green dot. The distance between the blue and green gives an indication of the amount of improvement.

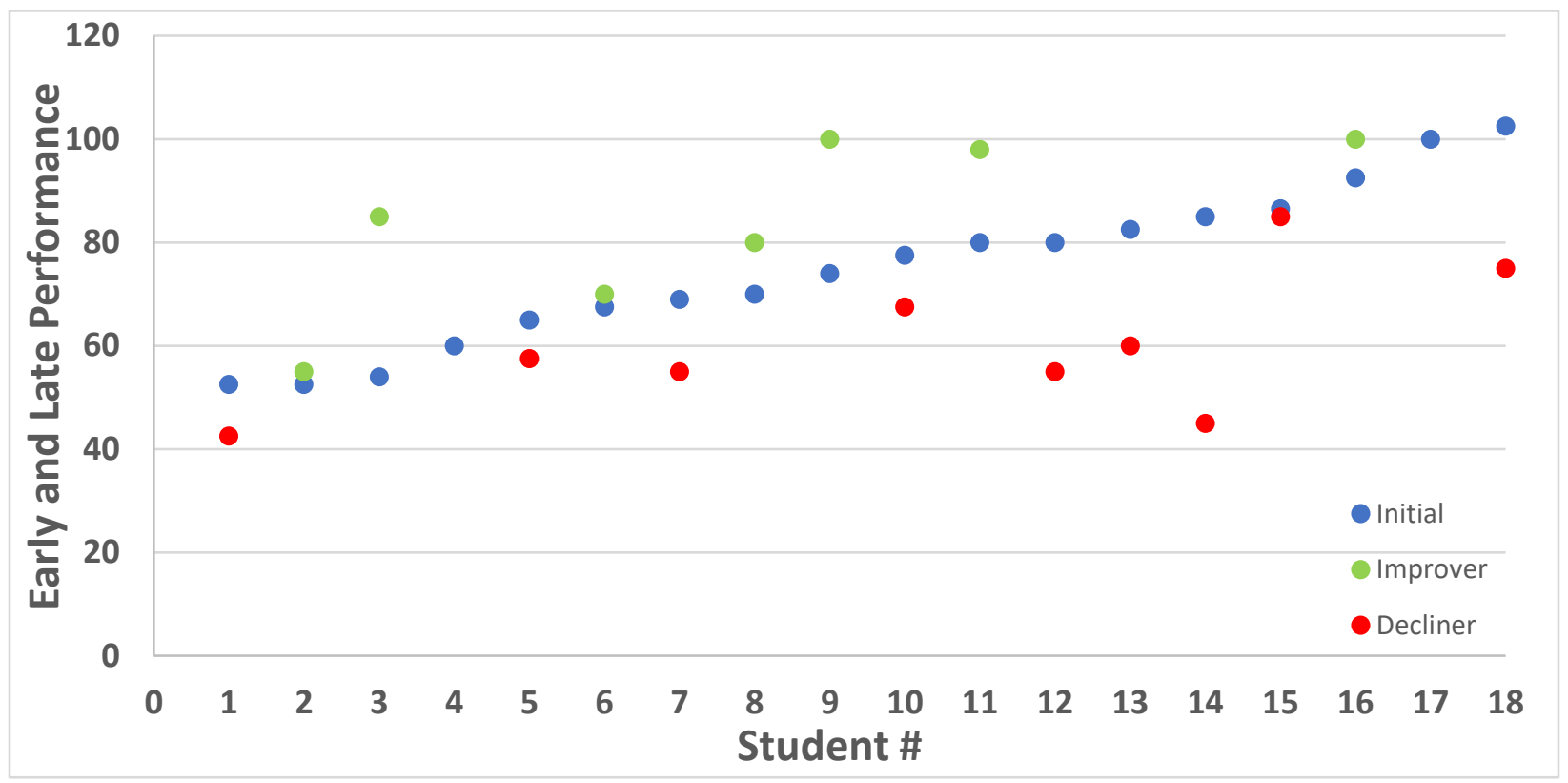

Figure 3 - Performance Change Per Inactive Student.

If the student's performance went down, the data shows a blue (initial) dot and a red dot. Notice that students 4 and 17 had no change. Note that 9 improved and 7 declined. This is some indication that the 4 exams were uniform. If the first two exams were easier or harder than the last two you would expect the majority of the students to either be improvers or decliners. Since it appears to be roughly equal, we believe the exams were well balanced. As indicated earlier, this is the data for inactive students and is being used to ascertain the difficulty level of the exams. Asymmetry in the projected data further corroborates the claims made by the authors.

Next, we plotted the performance change versus activity level. The data shown in Figure 4 is similar to the previous, the horizontal axis is increasing activity. Blue dots are initial grades and green and red dots indicate late performance improvers (green) and decliners (red). Note that the data is shown on a semi-log plot to help separate the students and make the data easier to see. To increase separation and make the plot easier to read we added increments of 0.01 to those with 0 activity. This causes the 0 activity students to spread out horizontally so they are visible.

Recognize that students with activities in the 100s are significantly more active than those in the 10 s due to the log plot.

There are a few interesting areas shown in the figure. Note that the most active students generally are improvers. Also note that the most active are not necessarily the beginning A students. It appears that most of the beginning A students have low activity, and many of them are decliners. Nearly all the beginning A students who are highly active are improvers. In fact, of the 22 students with activity greater than 10, only 4 are decliners. The cluster of decliners in the activity range of 1-10 shows that with limited activity profiles, students were unable to capitalize 
on the advantages of a collaborative study platform. As it is evident from the 10-100 range, the number of green dots (improvers) far exceed the red dots (decliners). This may also be due to the fact that students who started using the social media platform in the beginning were much more equipped and in sync with the classroom. When the opportunity for extra credit showed up, they used the momentum to further improve upon their grades in the class. 


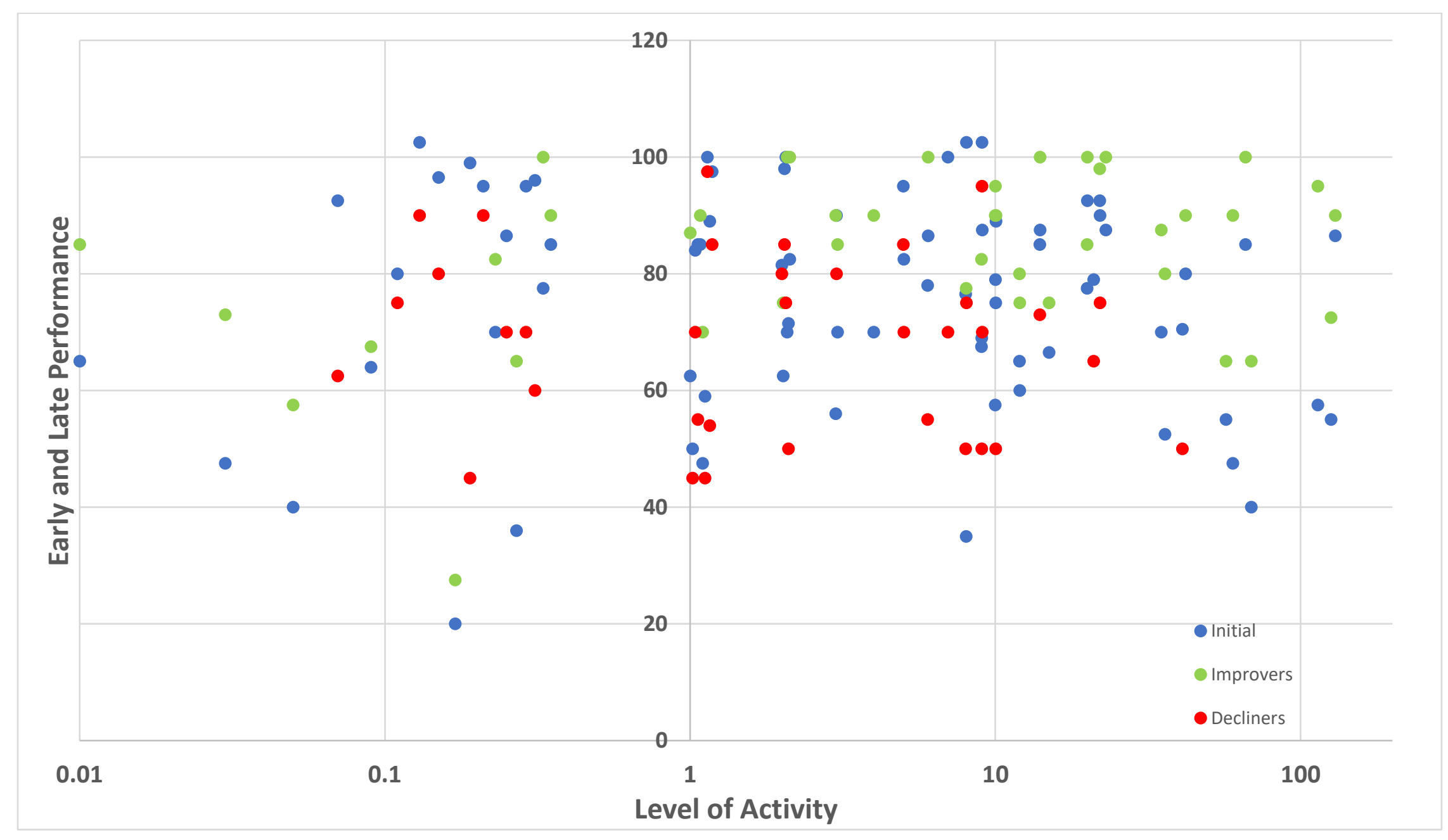

Figure 4 - Performance Change Versus Activity. 


\section{Conclusions:}

This experiment has attempted to quantify the impact social media software has on student performance. The data shows that some students exhibit instructor like behaviors of answering fellow student questions significantly more than posting questions. Future experiments intend to look for ways to encourage this behavior.

Data also demonstrated that as level of activity increases, students reached expected levels of engagement. Students who fell significantly short of the expected level of engagement were all in the low levels of activity. One interpretation of this is that students who are inactive are "out of touch" with the others in the class. This could be an indicator of students who are falling behind the class. Future experiments could focus on this question in more depth.

There were a number of students who had zero levels of activity. It is possible these students never logged on and therefore gained no benefit or handicap from the activities. It is also possible they logged on, read questions/answers and therefore benefitted from activity of others. Future work might track the time logged into the system to identify what the zero and low activity students are doing. Perhaps an exit survey at the end of the course could bring a clearer picture of this student behavior as well. This is reserved for future study.

Data showed that zero activity students had initial grades ranging from the high $\mathrm{F}$ to the high $\mathrm{A}$ range. Our initial expectation was the initial high-grade students might shy away from the activity. This would leave only the struggling students on-line trying to find help. Data shows this is not the case. Some of the most active students started with high grades. This is encouraging behavior because the stronger students are on-line and perhaps can provide better guidance for others.

Data showed that very highly active students had initial grades ranging from very low $\mathrm{F}$ to high A. Data also showed that those with the highest levels of activity ended up increasing their performance. Of the 22 students with activity levels greater than 10, 18 increased their grades and only 4 decreased. This indicates that there is a benefit to activity. All four decliners among the highly actives had initial grades from low B to low A. Three of the four active decliners passed the class, only one failed.

Finally, one should avoid drawing conclusions on the bulk of the students. For example, for the 77 students completing the study, 42 (55\%) improved their grades from the first two exams to the second two, and 35 declined indicating a modest number of students showing improvement. Studying the bulk however was not the purpose of the study. Recall that the purpose of the study was to determine if using the software produced significantly better performance. To see this you should compare the performance of light users of the software to heavy users. When looking at this comparison you can easily see from Figure 4, that of the 22 users with 10 or more Activity Level, $18(82 \%)$ were improvers. In contrast of the 55 users with less than 10 activity, only 24 (44\%) improved. Our conclusion is that the software helps students earn better grades if they use it in a significant way. 
References:

[1] Johnson, C., "A Survey of Current Research on Online Communities of Practice", The Internet and Higher Education, vol. 4, pp. 45-60, 2001.

[2] Wallace, R., "Online Learning in Higher Education: A Review of Research on Interactions Among Teachers and Students," Education Communication and Information, vol. 3, no. 2, pp. 241-280, July 2003.

[3] Rovai, A., and Jordan, H., "Blended Learning and Sense of Community: A Comparitive Analysis with Traditional and Fully Online Graduate Courses," International Review of Research in Open and Distance Learning, vol. 5, no. 2, pp. 1-13, August 2004.

[4] Williams, J., and Jacobs, J., "Exploring the Use of Blogs as Learning Spaces in the Higher Education Sector," Australian Journal of Educational Technology, vol. 20, no. 2, pp. 232-247, 2004.

[5] Davies, J., and Graff, M., "Performance in e-Learning: Online Participation and Student Grades," British Journal of Educational Technology, vol. 36, no. 4, pp. 657-663, 2005.

[6] Shea, P., and Bidjerano, T., "Learning Presence: Towards a Theory of Self-Efficacy SelfRegulation and the Development of a Communities of Inquiry in Online and Blended Leanring Environments," Computers and Education, vol. 55, pp. 1721-1731, 2010.

[7] Sadera, W., Robertson, J., Song, L., and Midon, N., "The Role of Community in Online Success," Journal of Online Learning and Teaching, vol. 5, no. 2, pp. 277-284, June 2009.

[8] Harrison, R., and Thomas, M., "Identity in Online Communities: Social Networking Sites and Language Learning," International Journal of Emerging Technologies and Society, vol. 7, no. 2, pp. 109-124, 2009.

[9] Brady, K., Holcomb, L., and Smith, B., "The Use of Alternative Social Networking Sites in Higher Educational Settings: A Case Study of the E-Learning Benfits of Ning in Education," Journal of Interactive Online Learning, vol. 9, no. 2, pp. 151-170, 2010.

[10] Hung, H., and Yuen, S., "Educational Use of Social Networking Technology in Higher Education," Teaching in Higher Education, vol. 15, no. 6, pp. 703-714, 2010.

[11] Chan, P. Graham-Day, K., Ressa, V., Peters, M., and Konrad, M., "Beyond Involvement: Promoting Student Ownership of Learning in Classrooms," Intervention in School and Clinic., vol. 50, no. 2, pp. 105-113, Nov. 2014.

[12] Schroeder, A., Minocha, S., and Schneider, C., "The Strengths, Weaknesses, Opportunities and Threats of Using Social Software in Higher and Further Education Teaching and Learning," Journal of Computer Assisted Learning., vol. 26, no. 3, pp. 159-174, 2010. 
[13] Tess, P., "The Role of Social Media in Higher Education Classes (Real and Virtual) - A Literature Review," Computers in Human Behavior., vol. 29, no. 5, pp. A60-A68, Sep. 2013. 